\title{
Microglial Responses after Ischemic Stroke and Intracerebral Hemorrhage
}

\author{
Roslyn A. Taylor ${ }^{1}$ and Lauren H. Sansing ${ }^{2,3,4}$ \\ ${ }^{1}$ Department of Immunology, University of Connecticut Health Center, Farmington, CT 06032, USA \\ ${ }^{2}$ Department of Neuroscience, University of Connecticut Health Center, Farmington, CT 06032, USA \\ ${ }^{3}$ Department of Neurology, University of Connecticut Health Center and Hartford Hospital, Hartford, CT 06102, USA \\ ${ }^{4}$ Department of Neurosurgery, Hartford Hospital, Hartford, CT 06102, USA
}

Correspondence should be addressed to Lauren H. Sansing; sansing@uchc.edu

Received 10 May 2013; Revised 6 August 2013; Accepted 28 August 2013

Academic Editor: Jeffrey Zirger

Copyright (C) 2013 R. A. Taylor and L. H. Sansing. This is an open access article distributed under the Creative Commons Attribution License, which permits unrestricted use, distribution, and reproduction in any medium, provided the original work is properly cited.

\begin{abstract}
Stroke is a leading cause of death worldwide. Ischemic stroke is caused by blockage of blood vessels in the brain leading to tissue death, while intracerebral hemorrhage (ICH) occurs when a blood vessel ruptures, exposing the brain to blood components. Both are associated with glial toxicity and neuroinflammation. Microglia, as the resident immune cells of the central nervous system (CNS), continually sample the environment for signs of injury and infection. Under homeostatic conditions, they have a ramified morphology and phagocytose debris. After stroke, microglia become activated, obtain an amoeboid morphology, and release inflammatory cytokines (the M1 phenotype). However, microglia can also be alternatively activated, performing crucial roles in limiting inflammation and phagocytosing tissue debris (the M2 phenotype). In rodent models, microglial activation occurs very early after stroke and $\mathrm{ICH}$; however, their specific roles in injury and repair remain unclear. This review summarizes the literature on microglial responses after ischemic stroke and $\mathrm{ICH}$, highlighting the mediators of microglial activation and potential therapeutic targets for each condition.
\end{abstract}

\section{Introduction}

Microglia are the resident immune cells of the central nervous system (CNS). The majority of our understanding about microglial responses to injury comes from rodent models. In mice, microglia arise from hematopoietic progenitors in the yolk sac at E8 in development [1]. Under normal physiological conditions, microglia self-renew and locally expand to maintain their numbers. However, blood-derived cells have been shown to regenerate microglial populations under scientific manipulations [2]. Microglia survey the CNS and phagocytose debris under homeostatic conditions as well as in injury and disease [3]. Microglia express Toll-like receptors (TLR) and NOD-like receptors (NLR), allowing them to detect bacterial pathogens and molecular signatures of injury, leading to the transcription of proinflammatory cytokine genes. Local [4] and systemic [5] infections, neurodegenerative conditions [6], and sterile injury [7] have been reported to activate microglia.

Once activated, microglia retract their ramifications and obtain an amoeboid morphology, becoming indistinguishable from activated macrophages. Both cell types derive from primitive myeloid cells, causing them to express many of the same markers (CD11b, F4/80, Iba-1) [1, 8], Due to this, studying microglial activity by immunohistochemistry has been difficult, causing researchers to identify activated phagocytes as microglia/macrophages. However, with the use of flow cytometry, populations of microglia and macrophages can be separated and studied individually due to their differences in CD45 expression [9]. Like macrophages, microglia can have either an M1, classically activated phenotype, or an M2, alternatively activated phenotype (Figure 1). M1 microglia are considered to be proinflammatory and secrete TNF- $\alpha$, iNOS, and CCL2. They express CD80, CD86, and MHCII on 


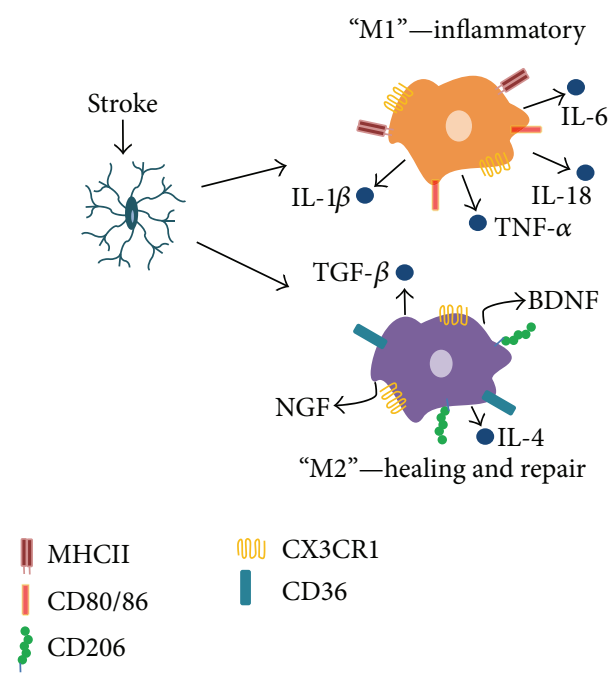

FIGURE 1: Microglia polarization is characterized by distinct phenotypes.

their cell surface, possessing the capability to present antigens to T cells [10]. They express IL-23, giving implications that microglia T-cell crosstalk may occur [11]. Microglia also produce IL- $1 \beta$ and IL-18 through activation of the inflammasome [11]. M2 microglia are interpreted to be healing cells that are involved with neuroprotection and repair after injury with arginase activity and upregulation of neurotrophic factors $[10,12]$. Because of their polarity, microglia have the potential to be both injurious and neuroprotective in CNS disease and injury. Here, we will review the role of microglia in neuroinflammation and acute injury after ischemic and hemorrhagic stroke.

\section{Cerebral Ischemia}

Stroke is the 4th leading cause of death in the United States, affecting 7 million people [13-15]. Ischemic stroke constitutes $87 \%$ of all strokes and is caused by the occlusion of a blood vessel due to either embolism or thrombus. As a result, brain tissue is deprived of blood glucose and oxygen [13]. This leads to neuronal death, release of reactive oxygen species, activation of complement, and upregulation of adhesion molecules on endothelial cells. Dying cells release danger signals into the environment, including HMGB1 and ATP, activating microglia [16]. This cascade of events leads to glial toxicity and infiltration of peripheral leukocytes $[14,16]$. The treatment for ischemic stroke is tissue plasminogen activator (tPA) which degrades the clot in the blood vessel in order to restore perfusion to the brain. However, tPA can only be administered to patients within a 3-to-4.5-hour window after the onset of stroke, and the majority of stroke patients are left with some infarction despite treatment $[17,18]$. With reperfusion of the brain or collateral circulation, peripheral leukocytes can infiltrate the brain to the area of injury and secrete proinflammatory cytokines, thus leading to secondary injury [14].

Microglial activation and proinflammatory cytokine production have been well characterized in rodent models of ischemic stroke. Early studies using flow cytometry show an increase in microglial populations in the ipsilateral hemisphere, whereas the contralateral hemisphere remains at basal levels [9]. As resident immune cells in the CNS, microglia are known to both phagocytose debris and secrete proinflammatory cytokines under ischemic conditions, contributing to tissue damage [19]. Under ischemic conditions, microglia can become destructive and phagocytose tissues as well. However, microglia have been reported to also secrete anti-inflammatory cytokines such as IL-10 and TGF$\beta$ [20-22], which act to quell inflammation. In studies where microglia have been ablated, mice had larger infarctions and a doubling of apoptotic neurons after ischemia, indicating the importance of microglial activity after ischemic stroke [23]. Thus, while microglia can be destructive in repair and recovery, their presence is needed to alleviate injury. The balance of these processes may depend on the location of the microglia, the degree of ischemia, and the timing after injury.

2.1. Microglial Activation in the Ischemic Core. The location of microglia in the ischemic brain changes their activation and cell fate. In the ischemic core, where blood flow is reduced to near zero, cell death is nearly universal by 24 hours [24]. In a 90-minute transient ischemia model, degenerating Ibal+ microglia are apparent 3.5-12 hours after reperfusion. Over 24-48 hours, round Ibal+ED1+ cells appear throughout the core [25]. Immediately after 60 minutes of focal ischemia without reperfusion, microglia/macrophages in the striatum (ischemic core) significantly increased the number of their processes. Twenty-four hours later, the microglia/macrophages in the ischemic core showed a reduction in numbers of processes, had significantly shorter processes, and increased CD11b expression indicating activation and the formation of an amoeboid morphology [24]. Twenty-four hours after permanent MCAO, few double positive $\mathrm{CD} 11 \mathrm{~b}+\mathrm{CD} 68^{+}$(a marker for phagocytosis) cells were found in the ischemic core; however, by day 7, CD68 expression increased. At 24 hours, M2 markers Ym1 and CD206 were exclusively found in the ischemic core, suggesting that the microglia/macrophages in the ischemic core promote tissue repair [26]. These findings were corroborated by another study examining the location of M1 versus M2 microglia/macrophages, which found M2 microglia/ macrophages infiltrating the ischemic core at 24 hours, peaking at 5 days, and declining in the striatum by 14 days [27]. In contrast, using the inflammatory marker CD16/32 ( $\mathrm{F} c \gamma$ receptors), they found $\mathrm{M} 1$ cells increasing in number in the striatum over time and outnumbering the M2 cells during the second week. However, nonvital (measurement of vitality undefined) cellular debris found in the ischemic core at 72 hours after photothrombosis in rats exhibited CD1lb staining, indicating that large numbers of microglia/macrophages in the ischemic core are destined to die [28]. Likewise, at 7 days after $\mathrm{TiO}_{2}$ sphere-medicated ischemia, CDllb+ cells in the ischemic core "showed signs of disintegration" [29]. These studies show that the microglia in the infarct core are initially injured as a result of ischemia. M2 microglia/macrophages then enter the area during the first week before declining 
in numbers, while M1 microglia/macrophages increase in numbers over the first 2 weeks.

2.2. Microglial Activation in the Peri-Infarct Zone. Microglia in the peri-infarct zone have different patterns of microglial activation than those found in the ischemic core. After 90 minutes of transient ischemia, activated Ibal+ED1- cells increased in number from 3.5 to 7 days after reperfusion but were decreased by day 14 [25]. In another study, eight and twenty-four hours after transient MCAO in mice, microglia/ macrophages in the border zone had shorter processes with fewer endpoints indicating activation. CD1lb and F4/80 expression were the greatest on those microglia/macrophages closest to the infarct border, localizing their activation to the site of injury $[24,30]$. Ym1 and CD206+ cells were not found in the peri-infarct zone at either 24 hours or 7 days in one study [26], while another found CD206+ cells in the cortex at the border zone of ischemia peaking at day 5 after ischemia before being outnumbered by M1 cells [27]. In both a photothrombosis model in rats and permanent MCAO in mice, the majority of the microglia/macrophages expressing CD68 were localized to the border zone and peri-infarct region [26, 28]. Microglia in the peri-infarct zone proliferated 48 and 72 hours after middle cerebral artery occlusion (MCAO); however, the amount of proliferation is reduced after 60-minute compared to 30-minute ischemia [31]. This work used CFSE and BrdU injection to distinguish between microglia and blood-derived macrophages histologically and determined that microglial proliferation, not macrophage recruitment, led to the increase in peri-infarct myeloid cells. By 72 hours after stroke, microglia/macrophages at the infarct border and peri-infarct region coexpress CD68 and MHCII, a marker of antigen presentation. By 7 days, the infarct was demarcated by a GFAP+ glial scar and double positive $\mathrm{CD}^{+} 8^{+}, \mathrm{MHCII}^{+}$phagocytic cells [32]. Together these studies indicate that the peri-infarct region is dominated by proinflammatory, proliferating, and activated microglia that increase in number over the first week after ischemia. These studies reveal that the dynamics of microglial phenotypes change over time and that the location of the microglia (core versus penumbra) is critical in determining that phenotype. However, these studies have been limited by an inability to differentiate between infiltrating macrophages and microglia, which both likely contributed to the measured phenotypes.

Bone marrow chimeras have been helpful in distinguishing the activated microglia from macrophages after ischemia. In this experimental paradigm, $\mathrm{C} 57 \mathrm{Bl} / 6$ mice were irradiated and the hematopoietic system reconstituted with bone marrow from mouse expressing GFP in leukocytes. Resident microglia, due to their radioresistant nature, would remain of host origin $\left(\mathrm{GFP}^{-}\right)$. Twenty-four hours after MCAO, activated, $\mathrm{GFP}^{-}$microglia were seen in the infarct region [33]. At 48 hours after stroke, GFP-Iba- $1^{+}$microglia ingested neuronal debris [34]. By 4 days after infarct, numerous $\mathrm{GFP}^{+}$, $\mathrm{F} 4 / 80^{+}$phagocytic cells were seen in the infarct area. These studies indicate that the activated, phagocytic $\mathrm{F} 4 / 80^{+}$cells identified in the early time points after ischemia are likely microglia $[33,34]$. While these studies help elucidate the role of microglia from monocytes after ischemia, it is important to note that radiation may alter the blood brain barrier. In a West Nile Virus infection model, bone marrow chimeras reconstituted with bone marrow from a $\mathrm{GFP}^{+}$mouse showed resting, ramified microglial-like cells which were $\mathrm{GFP}^{+}$, suggesting that blood-derived monocytes traffic into the brain after radiation and obtain a microglial morphology [35]. In order to properly study the role of microglia after stroke using bone marrow chimeras, heads need to be protected from radiation with a lead shield. Alternatively, a parabiosis model may be employed, although this precludes the ability to perform functional outcome testing.

\subsection{Microglial Cytokine Production after Ischemic Stroke.} Microglial cytokine production can be seen as early as 1 hour after stroke. Microglia have been reported to be the primary source of IL-1 $\beta$ in a biphasic time course peaking at 1 hour and 24 hours. IL-1 $\beta$ deficient mice have significantly smaller infarct volume 24 hours after permanent MCAO [36]. Microglia have also been reported to be the main source of IL-6, TGF- $\beta$, and IL-10 after ischemia [37]. RhIL-6 treatment 30 minutes prior to and 15 minutes after permanent MCAO notably reduced infarct size [38]. Within 4 hours after stroke, TNF- $\alpha$ production can be seen within and surrounding the infarct. Microglial/macrophage production of TNF- $\alpha$ can be measured 6, 12, and 24 hours after ischemic stroke [19]. However, the role of TNF- $\alpha$ production in ischemic stroke still remains controversial. While some studies found that TNF- $\alpha$ antagonism resulted in improved outcomes [39, 40], other studies found that TNF- $\alpha$ is important in hippocampal and striatal neurogenesis [40-42].

2.4. Mediators of Microglial Activation. The identification of specific mediators of microglial activation may provide a therapeutic target to alleviate microglial-mediated injury (Table 1). Recent work has identified galectin-3 as an important survival factor in microglial survival, proliferation, and migration $[23,43]$ after ischemic injury. In vivo, microglia/macrophages upregulate galectin- 372 hours after MCAO. In culture, WT microglia upregulate Iba-1, TLR2, CD68, and galectin-3. However, galectin $-3^{-/-}$microglia were only capable of upregulating Iba-1, indicating that galectin3 plays a role in the upregulation of TLR2 and CD68 [23]. The absence of galectin-3 led to larger areas of infarction and enhanced neuronal apoptosis after MCAO [23]. The role of Notch signaling has also been studied in microglial activation. Notch signaling occurs during inflammation and had been correlated to worse outcome after stroke. Transgenic mice with an antisense Notch have less CD11 staining; in the presence of LPS, microglia had less CD11b expression. In culture exposed to oxygen glucose deprivation (OGD), activated WT microglia upregulate Notch expression. Antisense Notch mice, when injected with LPS or subjected to MCAO, produced less TNF- $\alpha$ and IL- $1 \beta$ and also had attenuated $\mathrm{NF} \kappa \mathrm{B}$ p 65 activity, indicating that Notch signaling may play a role in microglia toxicity after ischemia [44]. These studies indicate that galectin-3 provides a mechanism for reducing cell death and infarction after cerebral ischemia, 
TABLE 1: Mediators of microglial activation after cerebral ischemia and intracerebral hemorrhage.

\begin{tabular}{|c|c|c|c|}
\hline Mediator & Measurements used & Results & Citation \\
\hline \multicolumn{4}{|c|}{ Ischemic stroke } \\
\hline Galectin 3 & $\begin{array}{l}\text { Galectin } 3^{-/-} \text {mice subjected to } 60 \text {-minute MCAO } \\
\text { followed by reperfusion for either } 24 \text { or } 72 \text { hours }\end{array}$ & $\begin{array}{l}\text { Galectin } 3 \text { reduces cell death and } \\
\text { infarct volume }\end{array}$ & {$[23]$} \\
\hline Notch & $\begin{array}{l}\text { Primary cell cultures and in vivo models of microglial } \\
\text { activation (LPS) and MCAO in antisense Notch mice }\end{array}$ & $\begin{array}{c}\text { Notch leads to increased } \\
\text { neuroinflammation }\end{array}$ & {$[44]$} \\
\hline SPARC & $\begin{array}{l}\text { Focal photothrombotic model of ischemic stroke in } \\
\qquad \text { SPARC }^{-/-} \text {mice }\end{array}$ & $\begin{array}{l}\mathrm{SPARC}^{-1-} \text { microglia have increased } \\
\text { processes length and branching and } \\
\text { increased microgliosis }\end{array}$ & {$[45]$} \\
\hline HMGB1 & $\begin{array}{c}\text { shRNA and HMGB1 inhibitor used to knock down } \\
\text { HMGB1 in ischemic stroke model and primary } \\
\text { microglial cultures }\end{array}$ & HMGB1 promotes neuroinflammation & {$[46-48]$} \\
\hline CX3CL1 & $\begin{array}{l}\text { Behavioral outcomes, edema, peripheral cell } \\
\text { infiltration, cytokine production in CX3CR1 knockout } \\
\text { mice in } 30 \text { - and } 60 \text {-minute MCAO }\end{array}$ & $\begin{array}{l}\text { CX3CL1-CX3CR1 signaling leads to } \\
\text { worse functional outcome and higher } \\
\text { neuroinflammation }\end{array}$ & {$[49-54]$} \\
\hline \multicolumn{4}{|c|}{$\begin{array}{l}\text { Intracerebral hemorrhage } \\
\end{array}$} \\
\hline Thrombin & $\begin{array}{l}\text { Thrombin injection in rats and in culture caused } \\
\text { neuronal apoptosis and increased cytokine production }\end{array}$ & $\begin{array}{l}\text { Activates microglia and promotes } \\
\text { cytokine production }\end{array}$ & [55-60] \\
\hline Heme & Blood or hemin injection & $\begin{array}{c}\text { Activates microglia and leads to } \\
\text { neuroinflammation }\end{array}$ & [61-63] \\
\hline
\end{tabular}

SPARC: secreted protein acidic rich in cysteine; HMGB1: high mobility group box 1.

while Notch signaling leads to enhanced inflammation and increased microglial neurotoxicity.

Secreted protein acidic rich in cysteine (SPARC) is a matricellular protein that regulates growth factors and the assembly of the extracellular matrix. SPARC has been shown to play a role in microgliosis after ischemia. Under homeostatic conditions, mature microglia express SPARC. In a focal photothrombotic cortical ischemic stroke model, microglia downregulate SPARC expression after injury. SPARC-null mice show increased processes length and branching at steady state in white matter. Microglial expansion is significantly increased in grey matter and reduced in white matter in the SPARC-null mice, indicating that SPARC plays a differential role in microglial expansion depending on the location in brain. By immunohistochemistry, SPARC-null mice had an increase in Gal-3 expression, indicating higher levels of microgliosis, which correlated to better functional outcome after cortical ischemia [45]. The evidence points to a role for SPARC in microglial toxicity and poor outcome after cerebral ischemia.

High motility group protein B1 (HMGB1) may act as a cytokine to activate microglia after ischemia [46]. HMGB1 increases in the blood and cerebral spinal fluid in rats after ischemia [46] and induces postischemia neurodegeneration [47]. When HMGB1 was reduced using a shRNA transgene injected into the striatum, the number of microglia in the infarct was reduced. Those in the infarct maintained a ramified morphology and had less p38 MAPK activity and TNF- $\alpha$, IL-1 $\beta, \mathrm{COX} 2$, and iNOS expression. In vitro, HMGB1 was released after incubation of microglial cultures with NMDAtreated cortical cells. Cultured microglia remained quiescent in this model when an HMGB1 inhibitor was introduced into the media with the cortical cells, identifying HMGB1 as the mediator of inflammation [46]. HMGB1 induces activation via the RAGE receptor on both microglia and blood-derived macrophages after ischemia [48]. Taken together, these studies indicate that HMGB1 may be a potential therapeutic target for modulating microglial activation and mediated injury.

The chemokine receptor CX3CR1 is expressed at high levels on murine microglia under homeostatic conditions. Its ligand, CX3CL1 (fractalkine), is produced by neurons and can be either secreted or membrane bound. When it is membrane-bound, neuronal CX3CL1 binds to microglial CX3CR1 and maintains microglia in a quiescent state $[64,65]$. Cleaved CX3CL1 acts as a chemokine to induce microglial chemotaxis [49]. The CX3CL1-CX3CR1 interaction has been shown to regulate microglial toxicity in models of Parkinson's, ALS, and LPS activation-when the receptor is nonfunctional, mice have worse functional outcome [65]. However, this neuroprotective role for microglial CX3CR1 signaling may not be preserved after ischemia. CX3CL1 expression is upregulated within 48 hours after MCAO and decreases by 7 days, while CX3CR1 expression was the highest on the microglia within the infarcted tissue at 7 days. These results suggest that this pathway may play a role in microglia/macrophage cell recruitment into the infarcted region [50]. However, mice genetically deficient for either CX3CR1 or CX3CL1 have smaller infarct volumes after MCAO [51-54]. This was accompanied by fewer blood-derived leukocytes infiltrating the brain at 72 hours and improved functional outcomes [53]. After 30 minutes of ischemia, $\mathrm{CX} 3 \mathrm{CR}^{-/-}$microglia remained in a ramified state, whereas WT microglia/macrophages had amoeboid morphology at 24 hours. WT microglia/macrophages had a proinflammatory profile with elevated iNOS and CD68 expression, whereas $\mathrm{CX} 3 \mathrm{CR}^{-/-}$microglia/macrophages had low expression of iNOS and CD68 but elevated Ym1 implicating a healing phenotype [52]. The addition of exogenous CX3CL1 intracerebroventricularly prior to MCAO in 
WT resulted in smaller infarct [54]. These studies indicate that global deficiency of this signaling pathway in both microglia and peripheral leukocytes is protective after ischemia. Through the use of bone marrow chimeras, the specific role of microglial CX3CR1 in infarct volume, functional outcome, and neuroinflammation can be better understood. It is possible that the differences observed in these studies were in part due to CX3CR1 deficiency on the peripheral monocytes. It has recently been reported that human bone marrow stromal cells transplanted into rats after MCAO used the fractalkine-CX3CR1 pathway to migrate to area of infarct [66]. In addition, in a clinical study, it was found that patients with lower concentrations of plasma CX3CL1 had worse outcome 6 months after stroke [67]. The apparently conflicting roles of CX3CL1-CX3CR1 signaling may be due to alterations in signaling pathways in genetically altered mice, opposing roles of this pathway on microglia and CX3CR1+ macrophages or other factors.

\subsection{Treatments Targeting Microglial Activation after Cerebral} Ischemia. While tPA is available to aid in reperfusion for selecting patients with ischemic strokes, there is a necessity for treatments to reduce injury and aid in repair after stroke. Microglial activation is one potential target. Several studies have investigated the role of minocycline on inhibiting or altering microglial activation. In a mouse model of ALS, minocycline attenuated microglial activation and reduced the expression of M1, but not M2, microglia/macrophage markers suggesting that minocycline inhibits the proinflammatory microglia/macrophages [68]. In mice, minocycline administered two hours after transient MCAO reduced infarct volume by $25 \%$ [69]. Rats which received continual minocycline treatment for 4 weeks after ischemia had reduced microglial activation by microscopy, which correlated with increased neurogenesis and better functional outcome [70]. Transplantation of bone marrow mononuclear cells (BMMC) is also being investigated as a possible treatment for ischemic stroke [71-73]. In vitro, BMMCs reduced neuronal death due to LPS and hypoxia activated mixed culture of microglia and peritoneal macrophages. Microglial cultures in the presence of BMMCs had higher levels of IL-10, VEGF, IGF1, and SDF1a [74]. Recent studies have investigated whether the addition of minocycline can improve functional outcome and neuroprotection after BMMC transfer after ischemia in vivo. Rats that received minocycline and BMMC treatment had reduced $\mathrm{CD} 8^{+}$cells by immunohistochemistry and better functional outcome $[75,76]$. These studies suggest that microglia contribute to neuroinflammation after ischemia and that BMMC therapy and minocycline have additive effects in reducing poststroke microglial activation. However, the dosing of minocycline is crucial for benefit; high doses induced toxicity in both neurons and astrocytes [77]. Minocycline is a tetracycline antibiotic that is not specific for microglia [77], and off-target effects may contribute to dose-limiting toxicities.

While many studies have focused on understanding the mechanism by which microglia cause secondary injury after stroke, other studies have shown that microglia are essential for prevention of neuronal apoptosis [23]. Microglia are needed for recovery and repair after ischemia. Rats transplanted with a human microglial cell line 48 hours after MCAO had better functional outcome 7,10 , and 14 days after stroke. The improved functional outcome correlated with fewer apoptotic cells, fewer CD68 phagocytic cells, and less GFAP glial scar in the ipsilateral hemisphere. Notably, rats that received human microglia had less endogenous microglial activation and upregulation of IL-4, IL-5, and neurotrophic factors, thus decreasing their neurotoxicity. Human microglial cells reduced ischemic injury and promoted repair in rats [78]. Therefore, microglia have potential for augmenting repair after ischemia and studies on the modulation of their phenotype towards healing processes may identify new therapeutic targets for stroke.

\section{Intracerebral Hemorrhage}

Research on the mechanisms of injury and repair after ICH has been more limited than after cerebral ischemia. ICH occurs when a blood vessel in the brain parenchyma ruptures, most commonly due to hypertension [79]. ICH has a high mortality rate: $30-50 \%$ of patients die within the first 30 days [79]. Of those who survive, only $20 \%$ regain independence within six months [80]. Despite recent advances in ICH research, no specific treatment for ICH currently exists [81]. The introduction of blood components, including thrombin, heme, and leukocytes and platelets, into the brain creates the basis for a secondary injury due to microglial activation and neuroinflammation resulting in the recruitment of leukocytes into a normally immune privileged site $[82,83]$.

The activation of microglia likely has dual roles after ICH. While some microglial processes may be beneficial, microglia have also been shown to play a role in the secondary injury that occurs after ICH [82]. A major role of microglial cells after ICH is to phagocytose the debris and erythrocytes left in the brain after hemorrhage. They have been shown to endocytose heme and hemoglobin. These processes are mediated through scavenger receptors, such as CD36, on their cell surface [84]. They also produce proinflammatory cytokines (TNF- $\alpha$, IL-1 $\beta$, IL-6) [85] and chemokines (CXCL2) [86], which promotes neuroinflammation and the recruitment of blood-derived leukocytes to the brain $[8,84]$.

3.1. Time Course of Microglial Activation after Intracerebral Hemorrhage. The activation of microglia/macrophages occurs early in the timeline of neuroinflammation following ICH. Microglial/macrophage activation within the perihematomal region was seen as early as 1 hour following ICH by immunofluorescence staining in the collagenase injection model of ICH and within 4 hours using the double injection method of whole blood [62, 87]. Microglial/macrophage production of IL-1 $\beta$ in rats can be seen as early as 6 hours and can persist up to 24 hours. Interestingly, there was no IL-6 or MMP-12 staining within the activated microglial/ macrophage population [88]. Twelve hours after a mouse model of ICH, microglial numbers between the ipsilateral and contralateral hemisphere did not differ, suggesting that robust proliferation or migration has not yet occurred [89]. However, by 72 hours microglia reach their peak number 
in the perihematomal region $[8,90]$, which corresponds to roughly a $40 \%$ increase in their numbers by flow cytometry [91]. A week after ICH, microglial/macrophage numbers begin to reduce; by 21 days, microglial/macrophage numbers have returned to basal levels, although some reports find that microglial/macrophage activation persists for 4 weeks $[8,90]$. A time course of functional study in rats has shown a correlation between the resolution of microglial/ macrophage numbers in the ipsilateral hemisphere with improvement in behavioral tests, suggesting that the presence of microglia/macrophages contributes to neurological deficit [90].

3.2. Mediators of Microglial Activation after ICH. Many studies have focused on the triggers of microglial activation after ICH. Blood components directly activate microglia and initiate immune responses. Thrombin, a serine protease in blood that is necessary for coagulation, causes apoptosis in neurons and astrocytes, provoking researchers to investigate whether thrombin plays a role in microglial activation after ICH [55]. In rats, direct injection of thrombin into the striatum caused neuronal apoptosis. Microglia upregulated CD11b expression and morphed from the ramified, resting state to an activated, amoeboid shape with increased p-ERK within 4 hours. By 24 hours, the activated microglia stained positive for iNOS and by 72 hours, the microglial/macrophage numbers in the ipsilateral striatum increased [56]. Thrombin-mediated activation of microglia is induced by MAPK signaling. Interestingly, MAPK inhibitors injected into the striatum prior to ICH caused a large reduction in pERK 8 hours after ICH and an increase of TUNEL positive microglia/macrophages [57]. It has also been reported that inhibitors of p38 MAPK and cJNK inhibitors not only caused microglial/macrophage apoptosis but also greatly reduced the TNF- $\alpha$ levels. These studies suggest that the MAPK pathway in microglia/macrophages is induced by thrombin and promotes cell maintenance, allowing the production of TNF [58].

The effect of thrombin on microglial proinflammatory cytokines and matrix metalloproteases (MMPs) has also been described. In culture, microglia express thrombin receptors and when stimulated with thrombin produce IL$1 \beta$ and TNF- $\alpha$. Cultures treated with tuftsin fragment1-3, a microglial/macrophage inhibitory factor (MIF), had less cytokine secretion. In vivo, mice treated with MIF had less edema, suggesting that activated microglia are a cause of blood-brain barrier dysfunction after ICH [59]. In vivo, thrombin promotes the cleavage of pro-MMP9 to active MMP9. $\mathrm{MMP9}^{-/-}$mice had less injury and microglial/ macrophage activation than wild-type mice [60]. Another work identified neutrophils as the main source of MMP9 [92]. Interestingly, neutrophil depletion did not change microglia numbers at 3 days after ICH [93] but was found to reduce microglial/macrophage populations seven days after ICH, as well as decrease the level of CD68 on microglial/macrophage cells 3 to 14 days after ICH [92].

Products of erythrocyte lysis, including heme and iron, are also active initiators of microglial activation and neuroinflammation. Heme is converted to ferric iron, biliverdin, and carbon monoxide by heme oxygenase (HO). Ironhandling proteins, including ferritin and hemosiderin, have been found within activated microglia/macrophages after ICH [59], suggesting that microglia are responsible for iron clearance and processing. Metalloporphyrin resulted in a decrease in ferritin deposition in microglia and less neuronal loss [61]. In aged rats, treatment with deferoxamine, an iron chelator, greatly reduced the number of activated microglia/ macrophages and the overall ROS production in the striatum [94]. Unconjugated bilirubin and bilirubin oxidative species are hypothesized to activate microglia in vivo, resulting in production of proinflammatory cytokines [95]. However, in a mouse animal model, unconjugated bilirubin infusion with the whole blood to create the $\mathrm{ICH}$, there was a reduction in microglia but increase in neutrophils at 24 hours [96]. The role of unconjugated bilirubin in microglial phenotype is yet unknown. Therapies modulating iron handling by microglia may improve outcomes by reducing both ironinduced oxidative damage and inflammation.

Toll-like receptor 4 (TLR4) activation also leads to neuroinflammation after ICH. In the CNS, microglia are the most prevailing cell type expressing TLR4 [97]. TLR4-deficient mice were shown to have reduced peripheral myeloid cell infiltration and fewer microglia in the perihematomal region 3 days after ICH, along with better functional outcome [91]. Another recent study found that heme degradation products lead to production of TNF- $\alpha$, IL-1 $\beta$, and IL- 6 via TLR4 in cultured microglia [63]; however, another work used blood transfer experiments to localize the location for TLR4 signaling to the cells in the ICH itself, rather than microglia [91]. Together, these studies indicate that TLR4 antagonism may be a potential therapeutic target for reducing microglial activation after ICH, either directly or by reducing leukocyte recruitment that then contributes to further microglial activation.

A recent study expanded upon HMGB1 acting as a proinflammatory cytokine after ICH. In vitro, heme stimulates cultured microglia to release HMGB1 [98]. After collagenaseinduced ICH in rats, the release of HMGB1 into the cytoplasm in the brain was detected by 1 hour. The administration of ethyl pyruvate reduced the number of $\mathrm{HGMBl}^{+}$cells in the ipsilateral hemisphere, improved functional outcome, and reduced edema and the number of apoptotic cells. Rats given ethyl pyruvate also had reduced numbers of activated microglia by immunohistochemistry and immunofluorescence [99]. As in ischemic stroke, targeting HMGB1 production after ICH may serve as a potential target to attenuate the immune response.

\subsection{Treatments Targeting Microglial Activation after Intracere-} bral Hemorrhage. While there is currently no treatment for microglial activation after $\mathrm{ICH}$, investigation into therapeutic targets is ongoing. Minocycline has been tested for neuroprotective qualities in ICH animal models as in ischemic stroke. One study administered minocycline to rats 3 hours after ICH to obtain clinical relevance. Minocycline had no effect on hemorrhage volume in either short term (5 days) or long term (28 days) survival but did reduce microglia/ 
macrophages numbers surrounding the hematoma at 5 days [100]. In other studies, animals were given minocycline treatment 6 hours, 1 day, and 2 days after ICH. Minocycline reduced the brain water content and increased intact blood vessels 72 hours after ICH. TNF- $\alpha$ and MMP12 levels were upregulated at 24 and 72 hours after $\mathrm{ICH}$, respectively. Minocycline treatment leads to a reduction in both TNF- $\alpha$ and MMP12 after ICH. However, the authors did not find colocalization of these proinflammatory factors with activated microglia/macrophages, but with neutrophils, suggesting that microglia may not be the only target of minocycline after ICH [101]. Thrombin-mediated activation of microglial cultures caused an upregulation in TNF- $\alpha$ and IL- $1 \beta$ production; minocycline treatment greatly reduced the production of both cytokines. In vivo, minocycline treatment reduced edema and improved functional outcome by 14 days [102]. Taken together, minocycline may serve as a promising treatment for ICH.

Peroxisome proliferator-activated receptor $\gamma(\operatorname{PPAR} \gamma)$ has also been investigated as a potential therapeutic for ICH. In mice, PPAR $\gamma$ agonist treatment beginning 24 hours after ICH enhanced the phagocytosis of the hematoma and reduced IL-1 $\beta$, TNF, MMP-9, and iNOS expression. In microglial cultures, PPAR $\gamma$ increased CD36-mediated microglial phagocytosis of red blood cells [103, 104]. Targeting microglial function (i.e., phagocytosis) as a therapeutic for ICH may have potential for modulating the immune response and enhancing recovery. Since recovery after ICH is at least partially dependent on microglial responses, therapies that modulate these responses towards repair have promise as treatments for $\mathrm{ICH}$.

\section{Conclusions}

Investigations on the role of microglia in the immune response after ischemic stroke and ICH can advance our understanding of the mechanisms of secondary injury and repair. Interestingly, the mediators of microglial activation differ between the two major types of strokes. In each condition, however, microglia can contribute to injury via the production of proinflammatory mediators and yet are crucial for remodeling and repair. Therapies that inhibit the injurious phase of microglial activation while augmenting repair would offer great promise for stroke patients. However, much of the work described above was performed on young, male rodents. The translational potential of the findings will be determined by the ability of therapies to improve outcomes across age, sex, and species. Future advances will also depend on differentiating the roles of microglia and macrophages in poststroke responses. With advances in scientific techniques, such as flow cytometry and cell sorting, the mechanisms by which microglia and macrophages contribute to neuroinflammation can be further understood, opening the possibility for new therapeutic targets.

\section{Acknowledgment}

This work was supported by K08NS078110 (LHS).

\section{References}

[1] F. Ginhoux, M. Greter, M. Leboeuf et al., "Fate mapping analysis reveals that adult microglia derive from primitive macrophages," Science, vol. 330, no. 6005, pp. 841-845, 2010.

[2] B. Ajami, J. L. Bennett, C. Krieger, W. Tetzlaff, and F. M. V. Rossi, "Local self-renewal can sustain CNS microglia maintenance and function throughout adult life," Nature Neuroscience, vol. 10, no. 12, pp. 1538-1543, 2007.

[3] U. Eyo and M. Dailey, "Microglia: key elements in neural development, plasticity, and pathology," Journal of Neuroimmune Pharmacology, vol. 8, no. 3, pp. 494-509, 2013.

[4] Y. Suzuki, Q. Sa, M. Gehman, and E. Ochiai, "Interferongamma- and perforin-mediated immune responses for resistance against Toxoplasma gondii in the brain," Expert reviews in molecular medicine, vol. 13, p. e31, 2011.

[5] U. Puntener, S. G. Booth, V. H. Perry, and J. L. Teeling, "Longterm impact of systemic bacterial infection on the cerebral vasculature and microglia," Journal of Neuroinflammation, vol. 9, p. 146, 2012.

[6] T. C. Browne, K. McQuillan, R. M. McManus, J. A. O. 'Reilly, K. H. Mills, and M. A. Lynch, "IFN- $\gamma$ production by amyloid $\beta$ specific Th1 cells promotes microglial activation and increases plaque burden in a mouse model of Alzheimer's disease," The Journal of Immunology, vol. 190, no. 5, pp. 2241-2251, 2013.

[7] N. Imamoto, S. Momosaki, M. Fujita et al., "[11C]PK11195 PET imaging of spinal glial activation after nerve injury in rats," Neuroimage, vol. 79, pp. 121-128, 2013.

[8] J. Wang, "Preclinical and clinical research on inflammation after intracerebral hemorrhage," Progress in Neurobiology, vol. 92, no. 4, pp. 463-477, 2010.

[9] M. Campanella, C. Sciorati, G. Tarozzo, and M. Beltramo, "Flow cytometric analysis of inflammatory cells in ischemic rat brain," Stroke, vol. 33, no. 2, pp. 586-592, 2002.

[10] S. Starossom, I. Mascanfroni, J. Imitola et al., "Galectin-1 deactivates classically activated microglia and protects from inflammation-induced neurodegeneration," Immunity, vol. 37, no. 2, pp. 249-263, 2012.

[11] R. M. Ransohoff and M. A. Brown, "Innate immunity in the central nervous system," Journal of Clinical Investigation, vol. 122, no. 4, pp. 1164-1171, 2012.

[12] J. Kawanokuchi, K. Shimizu, A. Nitta et al., "Production and functions of IL-17 in microglia," Journal of Neuroimmunology, vol. 194, no. 1-2, pp. 54-61, 2008.

[13] M. Zhou, C. Wang, W. L. Yang, and P. Wang, "Microglial CD14 activated by iNOS contributes to neuroinflammation in cerebral ischemia," Brain Research, vol. 1506, pp. 105-114, 2013.

[14] I. Onwuekwe and B. Ezeala-Adikaibe, "Ischemic stroke and neuroprotection," Annals of Medical and Health Sciences Research, vol. 2, no. 2, pp. 186-190, 2012.

[15] A. Towfighi and J. L. Saver, "Stroke declines from third to fourth leading cause of death in the United States: historical perspective and challenges ahead," Stroke, vol. 42, no. 8, pp. 2351-2355, 2011.

[16] C. Iadecola and J. Anrather, "The immunology of stroke: from mechanisms to translation," Nature Medicine, vol. 17, no. 7, pp. 796-808, 2011.

[17] S. Savitz and H. Mattle, "Advances in stroke: emerging therapies," Stroke, vol. 44, no. 2, pp. 314-315, 2013.

[18] X. Urra and A. Chamorro, "Emerging issues in acute ischemic stroke," Journal of Neurology, vol. 260, no. 6, pp. 1687-1692, 2013. 
[19] R. Gregersen, K. Lambertsen, and B. Finsen, "Microglia and macrophages are the major source of tumor necrosis factor in permanent middle cerebral artery occlusion in mice," Journal of Cerebral Blood Flow and Metabolism, vol. 20, no. 1, pp. 53-65, 2000.

[20] E. Parada, J. Egea, I. Buendia et al., "The Microglial alpha7acetylcholine nicotinic receptor is a key element in promoting neuroprotection by inducing heme oxygenase-1 via nuclear factor erythroid-2-related factor 2," Antioxidants \& Redox Signaling, vol. 19, no. 11, pp. 1135-1148, 2013.

[21] E. Lehrmann, R. Kiefer, T. Christensen et al., "Microglia and macrophages are major sources of locally produced transforming growth factor-betal after transient middle cerebral artery occlusion in rats," Glia, vol. 24, no. 4, pp. 437-448, 1998.

[22] F. De Bilbao, D. Arsenijevic, T. Moll et al., "In vivo overexpression of interleukin-10 increases resistance to focal brain ischemia in mice," Journal of Neurochemistry, vol. 110, no. 1, pp. 12-22, 2009.

[23] M. Lalancette-Hébert, V. Swarup, J. Beaulieu et al., "Galectin3 is required for resident microglia activation and proliferation in response to ischemic injury," The Journal of Neuroscience, vol. 32, no. 30, pp. 10383-10395, 2012.

[24] H. W. Morrison and J. A. Filosa, "A quantitative spatiotemporal analysis of microglia morphology during ischemic stroke and reperfusion," Journal of Neuroinflammation, vol. 10, p. 4, 2013.

[25] D. Ito, K. Tanaka, S. Suzuki, T. Dembo, and Y. Fukuuchi, "Enhanced expression of Ibal, ionized calcium-binding adapter molecule 1, after transient focal cerebral ischemia in rat brain," Stroke, vol. 32, no. 5, pp. 1208-1215, 2001.

[26] C. Perego, S. Fumagalli, and M.-G. De Simoni, “Temporal pattern of expression and colocalization of microglia/macrophage phenotype markers following brain ischemic injury in mice," Journal of Neuroinflammation, vol. 8, article 174, 2011.

[27] X. Hu, P. Li, Y. Guo et al., "Microglia/macrophage polarization dynamics reveal novel mechanism of injury expansion after focal cerebral ischemia," Stroke, vol. 43, no. 11, pp. 3063-3070, 2012.

[28] M. Schroeter, S. Jander, O. W. Witte, and G. Stoll, "Heterogeneity of the microglial response in photochemically induced focal ischemia of the rat cerebral cortex," Neuroscience, vol. 89, no. 4, pp. 1367-1377, 1999.

[29] M. Schroeter, M. A. Dennin, M. Walberer et al., "Neuroinflammation extends brain tissue at risk to vital peri-infarct tissue: a double tracer [11C]PK11195- and [18F]FDG-PET study," Journal of Cerebral Blood Flow and Metabolism, vol. 29, no. 6, pp. 12161225, 2009.

[30] M. Wiart, N. Davoust, J.-B. Pialat et al., "MRI monitoring of neuroinflammation in mouse focal ischemia," Stroke, vol. 38, no. 1, pp. 131-137, 2007.

[31] A. Denes, R. Vidyasagar, J. Feng et al., "Proliferating resident microglia after focal cerebral ischaemia in mice," Journal of Cerebral Blood Flow and Metabolism, vol. 27, no. 12, pp. 19411953, 2007.

[32] M. Walberer, M. A. Rueger, M.-L. Simard et al., "Dynamics of neuroinflammation in the macrosphere model of arterio-arterial embolic focal ischemia: an approximation to human stroke patterns," Experimental and Translational Stroke Medicine, vol. 2, no. 1, article 22, 2010.

[33] M. Schilling, M. Besselmann, C. Leonhard, M. Mueller, E. B. Ringelstein, and R. Kiefer, "Microglial activation precedes and predominates over macrophage infiltration in transient focal cerebral ischemia: a study in green fluorescent protein transgenic bone marrow chimeric mice," Experimental Neurology, vol. 183, no. 1, pp. 25-33, 2003.

[34] M. Schilling, M. Besselmann, M. Müller, J. K. Strecker, E. B. Ringelstein, and R. Kiefer, "Predominant phagocytic activity of resident microglia over hematogenous macrophages following transient focal cerebral ischemia: an investigation using green fluorescent protein transgenic bone marrow chimeric mice," Experimental Neurology, vol. 196, no. 2, pp. 290-297, 2005.

[35] D. R. Getts, R. L. Terry, M. T. Getts et al., "Ly6c "inflammatory monocytes" are microglial precursors recruited in a pathogenic manner in West Nile virus encephalitis," Journal of Experimental Medicine, vol. 205, no. 10, pp. 2319-2337, 2008.

[36] G. P. Schielke, G.-Y. Yang, B. D. Shivers, and A. L. Betz, "Reduced ischemic brain injury in interleukin- $1 \beta$ converting enzyme-deficient mice," Journal of Cerebral Blood Flow and Metabolism, vol. 18, no. 2, pp. 180-185, 1998.

[37] A.-G. Ceulemans, T. Zgavc, R. Kooijman, S. Hachimi-Idrissi, S. Sarre, and Y. Michotte, "The dual role of the neuroinflammatory response after ischemic stroke: modulatory effects of hypothermia," Journal of Neuroinflammation, vol. 7, article 74, 2010.

[38] S. A. Loddick, A. V. Turnbull, and N. J. Rothwell, "Cerebral interleukin-6 is neuroprotective during permanent focal cerebral ischemia in the rat," Journal of Cerebral Blood Flow and Metabolism, vol. 18, no. 2, pp. 176-179, 1998.

[39] X. Wang, G. Z. Feuerstein, L. Xu et al., "Inhibition of tumor necrosis factor- $\alpha$-converting enzyme by a selective antagonist protects brain from focal ischemic injury in rats," Molecular Pharmacology, vol. 65, no. 4, pp. 890-896, 2004.

[40] M. K. McCoy and M. G. Tansey, “TNF signaling inhibition in the CNS: implications for normal brain function and neurodegenerative disease," Journal of Neuroinflammation, vol. 5, article 45, 2008.

[41] U. Heldmann, P. Thored, J.-H. Claasen, A. Arvidsson, Z. Kokaia, and O. Lindvall, "TNF- $\alpha$ antibody infusion impairs survival of stroke-generated neuroblasts in adult rat brain," Experimental Neurology, vol. 196, no. 1, pp. 204-208, 2005.

[42] K. L. Lambertsen, B. H. Clausen, A. A. Babcock et al., "Microglia protect neurons against ischemia by synthesis of tumor necrosis factor," Journal of Neuroscience, vol. 29, no. 5, pp. 1319-1330, 2009.

[43] U. Wesley, R. Vemuganti, E. Ayvaci, and R. Dempsey, "Galectin3 enhances angiogenic and migratory potential of microglial cells via modulation of integrin linked kinase signaling," Brain Research, vol. 1496, pp. 1-9, 2013.

[44] Z. Wei, S. Chigurupati, T. V. Arumugam, D.-G. Jo, H. Li, and S. L. Chan, "Notch activation enhances the microglia-mediated inflammatory response associated with focal cerebral ischemia," Stroke, vol. 42, no. 9, pp. 2589-2594, 2011.

[45] S. M. Lloyd-Burton, E. M. York, M. A. Anwar, A. J. Vincent, and A. J. Roskams, "SPARC regulates microgliosis and functional recovery following cortical ischemia," The Journal of Neuroscience, vol. 33, no. 10, pp. 4468-4481, 2013.

[46] J.-B. Kim, S. C. Joon, Y.-M. Yu et al., "HMGB1, a novel cytokinelike mediator linking acute neuronal death and delayed neuroinflammation in the postischemic brain," Journal of Neuroscience, vol. 26, no. 24, pp. 6413-6421, 2006.

[47] G. Faraco, S. Fossati, M. E. Bianchi et al., "High mobility group box 1 protein is released by neural cells upon different stresses and worsens ischemic neurodegeneration in vitro and in vivo," Journal of Neurochemistry, vol. 103, no. 2, pp. 590-603, 2007. 
[48] S. Muhammad, W. Barakat, S. Stoyanov et al., "The HMGB1 receptor RAGE mediates ischemic brain damage," Journal of Neuroscience, vol. 28, no. 46, pp. 12023-12031, 2008.

[49] G. A. Chapman, K. Moores, D. Harrison, C. A. Campbell, B. R. Stewart, and P. J. Strijbos, "Fractalkine cleavage from neuronal membranes represents an acute event in the inflammatory response to excitotoxic brain damage," Journal of Neuroscience, vol. 20, no. 15, p. RC87, 2000.

[50] G. Tarozzo, M. Campanella, M. Ghiani, A. Bulfone, and M. Beltramo, "Expression of fractalkine and its receptor, CX3CR1, in response to ischaemia-reperfusion brain injury in the rat," European Journal of Neuroscience, vol. 15, no. 10, pp. 1663-1668, 2002.

[51] S. G. Soriano, L. S. Amaravadi, Y. F. Wang et al., "Mice deficient in fractalkine are less susceptible to cerebral ischemiareperfusion injury," Journal of Neuroimmunology, vol. 125, no. 1-2, pp. 59-65, 2002.

[52] S. Fumagalli, C. Perego, F. Ortolano, and M. G. De Simoni, "CX3CR1 deficiency induces an early protective inflammatory environment in ischemic mice," Glia, vol. 61, no. 6, pp. 827-842, 2013.

[53] Á. Dénes, S. Ferenczi, J. Halász, Z. Környei, and K. J. Kovács, "Role of CX3CR1 (fractalkine receptor) in brain damage and inflammation induced by focal cerebral ischemia in mouse," Journal of Cerebral Blood Flow and Metabolism, vol. 28, no. 10, pp. 1707-1721, 2008.

[54] R. Cipriani, P. Villa, G. Chece et al., "CX3CL1 is neuroprotective in permanent focal cerebral ischemia in rodents," Journal of Neuroscience, vol. 31, no. 45, pp. 16327-16335, 2011.

[55] F. Donovan, C. Pike, C. Cotman, and D. D. Cunningham, "Thrombin induces apoptosis in cultured neurons and astrocytes via a pathway requiring tyrosine kinase and RhoA activities," Journal of Neuroscience, vol. 17, no. 14, pp. 5316-5326, 1997.

[56] S. Fujimoto, H. Katsuki, M. Ohnishi, M. Takagi, T. Kume, and A. Akaike, "Thrombin induces striatal neurotoxicity depending on mitogen-activated protein kinase pathways in vivo," Neuroscience, vol. 144, no. 2, pp. 694-701, 2007.

[57] M. Ohnishi, H. Katsuki, S. Fujimoto, M. Takagi, T. Kume, and A. Akaike, "Involvement of thrombin and mitogen-activated protein kinase pathways in hemorrhagic brain injury," Experimental Neurology, vol. 206, no. 1, pp. 43-52, 2007.

[58] M. Ohnishi, H. Katsuki, Y. Izumi, T. Kume, Y. Takada-Takatori, and A. Akaike, "Mitogen-activated protein kinases support survival of activated microglia that mediate thrombin-induced striatal injury in organotypic slice culture," Journal of Neuroscience Research, vol. 88, no. 10, pp. 2155-2164, 2010.

[59] J. Wu, S. Yang, G. Xi et al., "Microglial activation and brain injury after intracerebral hemorrhage," Acta Neurochirurgica, Supplementum, no. 105, pp. 59-65, 2008.

[60] M. Xue, M. D. Hollenberg, and V. W. Yong, "Combination of thrombin and matrix metalloproteinase- 9 exacerbates neurotoxicity in cell culture and intracerebral hemorrhage in mice," Journal of Neuroscience, vol. 26, no. 40, pp. 10281-10291, 2006.

[61] A. H. Koeppen, A. C. Dickson, and J. Smith, "Heme oxygenase in experimental intracerebral hemorrhage: the benefit of tinmesoporphyrin," Journal of Neuropathology and Experimental Neurology, vol. 63, no. 6, pp. 587-597, 2004.

[62] J. Wang and S. Doré, "Heme oxygenase-1 exacerbates early brain injury after intracerebral haemorrhage," Brain, vol. 130, no. 6, pp. 1643-1652, 2007.

[63] S. Lin, Q. Yin, Q. Zhong et al., "Heme activates TLR4-mediated inflammatory injury via MyD88/TRIF signaling pathway in intracerebral hemorrhage," Journal of Neuroinflammation, vol. 9, article 46, 2012.

[64] J. K. Harrison, Y. Jiang, S. Chen et al., "Role for neuronally derived fractalkine in mediating interactions between neurons and CX3CR1-expressing microglia," Proceedings of the National Academy of Sciences of the United States of America, vol. 95, no. 18, pp. 10896-10901, 1998.

[65] A. E. Cardona, E. P. Pioro, M. E. Sasse et al., "Control of microglial neurotoxicity by the fractalkine receptor," Nature Neuroscience, vol. 9, no. 7, pp. 917-924, 2006.

[66] J. Zhu, Z. Zhou, Y. Liu, and J. Zheng, "Fractalkine and CX3CR1 are involved in the migration of intravenously grafted human bone marrow stromal cells toward ischemic brain lesion in rats," Brain Research, vol. 1287, pp. 173-183, 2009.

[67] M. M. Donohue, K. Cain, D. Zierath, D. Shibata, P. M. Tanzi, and K. J. Becker, "Higher plasma fractalkine is associated with better 6-month outcome from ischemic stroke," Stroke, vol. 43, no. 9, pp. 2300-2306, 2012.

[68] K. Kobayashi, S. Imagama, T. Ohgomori et al., "Minocycline selectively inhibits M1 polarization of microglia," Cell Death and Disease, vol. 4, p. e525, 2013.

[69] Y. C. Weng and J. Kriz, "Differential neuroprotective effects of a minocycline-based drug cocktail in transient and permanent focal cerebral ischemia," Experimental Neurology, vol. 204, no. 1, pp. 433-442, 2007.

[70] Z. Liu, Y. Fan, S. J. Won et al., "Chronic treatment with minocycline preserves adult new neurons and reduces functional impairment after focal cerebral ischemia," Stroke, vol. 38, no. 1, pp. 146-152, 2007.

[71] M. Brenneman, S. Sharma, M. Harting et al., "Autologous bone marrow mononuclear cells enhance recovery after acute ischemic stroke in young and middle-aged rats," Journal of Cerebral Blood Flow and Metabolism, vol. 30, no. 1, pp. 140-149, 2010.

[72] E. Keimpema, M. R. Fokkens, Z. Nagy et al., "Early transient presence of implanted bone marrow stem cells reduces lesion size after cerebral ischaemia in adult rats," Neuropathology and Applied Neurobiology, vol. 35, no. 1, pp. 89-102, 2009.

[73] A. de Vasconcelos dos Santos, J. da Costa Reis, B. Diaz Paredes et al., "Therapeutic window for treatment of cortical ischemia with bone marrow-derived cells in rats," Brain Research, vol. 1306, pp. 149-158, 2010.

[74] S. Sharma, B. Yang, R. Strong et al., "Bone marrow mononuclear cells protect neurons and modulate microglia in cell culture models of ischemic stroke," Journal of Neuroscience Research, vol. 88, no. 13, pp. 2869-2876, 2010.

[75] M. Cardoso, E. Franco, C. de Souza, M. da Silva, A. Gouveia, and W. Gomes-Leal, "Minocycline treatment and bone marrow mononuclear cell transplantation after endothelin-1 induced striatal ischemia," Inflammation, vol. 36, no. 1, pp. 197-205, 2013.

[76] E. C. S. Franco, M. M. Cardoso, A. Gouvêia, A. Pereira, and W. Gomes-Leal, "Modulation of microglial activation enhances neuroprotection and functional recovery derived from bone marrow mononuclear cell transplantation after cortical ischemia," Neuroscience Research, vol. 73, no. 2, pp. 122-132, 2012.

[77] N. Matsukawa, T. Yasuhara, K. Hara et al., "Therapeutic targets and limits of minocycline neuroprotection in experimental ischemic stroke," BMC Neuroscience, vol. 10, article 1471, p. 126, 2009.

[78] D. Narantuya, A. Nagai, M. Abdullah et al., "Human microglia transplanted in rat focal ischemia brain induce neuroprotection 
and behavioral improvement," PLoS ONE, vol. 5, no. 7, Article ID el1746, 2010.

[79] E. Manno, "Update on intracerebral hemorrhage," Continuum, vol. 18, no. 3, pp. 598-610, 2012.

[80] A. I. Qureshi, A. D. Mendelow, and D. F. Hanley, "Intracerebral haemorrhage," The Lancet, vol. 373, no. 9675, pp. 1632-1644, 2009.

[81] L. B. Morgenstern, J. C. Hemphill III, C. Anderson et al., "Guidelines for the management of spontaneous intracerebral hemorrhage: a guideline for healthcare professionals from the American Heart Association/American Stroke Association," Stroke, vol. 41, no. 9, pp. 2108-2129, 2010.

[82] R. Keep, Y. Hua, and G. Xi, "Intracerebral haemorrhage: mechanisms of injury and therapeutic targets," Lancet Neurology, vol. 11, no. 8, pp. 720-731, 2012.

[83] M. J. Carson, J. M. Doose, B. Melchior, C. D. Schmid, and C. C. Ploix, "CNS immune privilege: hiding in plain sight," Immunological Reviews, vol. 213, no. 1, pp. 48-65, 2006.

[84] J. Aronowski and X. Zhao, "Molecular pathophysiology of cerebral hemorrhage: secondary brain injury," Stroke, vol. 42, no. 6, pp. 1781-1786, 2011.

[85] J. Wang and S. Doré, "Inflammation after intracerebral hemorrhage," Journal of Cerebral Blood Flow and Metabolism, vol. 27, no. 5, pp. 894-908, 2007.

[86] D. B. Kurland, V. Gerzanich, and J. M. Simard, "191 Heme induces microglial CXCL2 release-a mechanism of neutrophilmediated injury after intracerebral hemorrhage," Neurosurgery, vol. 60, supplement 1, p. 183, 2013.

[87] M. Xue and M. R. Del Bigio, "Intracerebral injection of autologous whole blood in rats: time course of inflammation and cell death," Neuroscience Letters, vol. 283, no. 3, pp. 230-232, 2000.

[88] J. K. Wasserman, X. Zhu, and L. C. Schlichter, "Evolution of the inflammatory response in the brain following intracerebral hemorrhage and effects of delayed minocycline treatment," Brain Research, vol. 1180, no. 1, pp. 140-154, 2007.

[89] M. D. Hammond, Y. Ai, and L. H. Sansing, "Gr1 ${ }^{+}$macrophages and dendritic cells dominate the inflammatory infiltrate 12 $\mathrm{h}$ after experimental intracerebral hemorrhage," Translational Stroke Research, vol. 3, 1, pp. 125-131, 2012.

[90] A. Yabluchanskiy, P. Sawle, S. Homer-Vanniasinkam, C. J. Green, and R. Motterlini, "Relationship between leukocyte kinetics and behavioral tests changes in the inflammatory process of hemorrhagic stroke recovery," International Journal of Neuroscience, vol. 120, no. 12, pp. 765-773, 2010.

[91] L. H. Sansing, T. H. Harris, F. A. Welsh, S. E. Kasner, C. A. Hunter, and K. Kariko, "Toll-like receptor 4 contributes to poor outcome after intracerebral hemorrhage," Annals of Neurology, vol. 70, no. 4, pp. 646-656, 2011.

[92] I. Moxon-Emre and L. C. Schlichter, "Neutrophil depletion reduces blood-brain barrier breakdown, axon injury, and inflammation after intracerebral hemorrhage," Journal of Neuropathology and Experimental Neurology, vol. 70, no. 3, pp. 218235, 2011.

[93] L. H. Sansing, T. H. Harris, S. E. Kasner, C. A. Hunter, and K. Kariko, "Neutrophil depletion diminishes monocyte infiltration and improves functional outcome after experimental intracerebral hemorrhage," Acta Neurochirurgica, Supplementum, no. 111, pp. 173-178, 2011.

[94] H. Wu, T. Wu, X. Xu, J. Wang, and J. Wang, "Iron toxicity in mice with collagenase-induced intracerebral hemorrhage," Journal of Cerebral Blood Flow and Metabolism, vol. 31, no. 5, pp. 12431250, 2011.
[95] M. C. Loftspring, C. Hansen, and J. F. Clark, "A novel brain injury mechanism after intracerebral hemorrhage: the interaction between heme products and the immune system," Medical Hypotheses, vol. 74, no. 1, pp. 63-66, 2010.

[96] M. C. Loftspring, H. L. Johnson, R. Feng, A. J. Johnson, and J. F. Clark, "Unconjugated bilirubin contributes to early inflammation and edema after intracerebral hemorrhage," Journal of Cerebral Blood Flow and Metabolism, vol. 31, no. 4, pp.1133-1142, 2011.

[97] H. Fang, P. F. Wang, Y. Zhou, Y. C. Wang, and Q. W. Yang, “Tolllike receptor 4 signaling in intracerebral hemorrhage-induced inflammation and injury," Journal of Neuroinflammation, vol. 10, p. 27, 2013.

[98] Y. Zhou, K.-L. Xiong, S. Lin et al., "Elevation of high-mobility group protein box-1 in serum correlates with severity of acute intracerebral hemorrhage," Mediators of Inflammation, vol. 2010, Article ID 142458, 6 pages, 2010.

[99] C. Lei, S. Lin, C. Zhang et al., "High-mobility group box1 protein promotes neuroinflammation after intracerebral hemorrhage in rats," Neuroscience, vol. 228, pp. 190-199, 2013.

[100] A. Szymanska, J. Biernaskie, D. Laidley, S. Granter-Button, and D. Corbett, "Minocycline and intracerebral hemorrhage: influence of injury severity and delay to treatment," Experimental Neurology, vol. 197, no. 1, pp. 189-196, 2006.

[101] J. K. Wasserman and L. C. Schlichter, "Minocycline protects the blood-brain barrier and reduces edema following intracerebral hemorrhage in the rat," Experimental Neurology, vol. 207, no. 2, pp. 227-237, 2007.

[102] J. Wu, S. Yang, G. Xi, G. Fu, R. F. Keep, and Y. Hua, "Minocycline reduces intracerebral hemorrhage-induced brain injury," Neurological Research, vol. 31, no. 2, pp. 183-188, 2009.

[103] X. Zhao, G. Sun, J. Zhang et al., "Hematoma resolution as a target for intracerebral hemorrhage treatment: role for peroxisome proliferator-activated receptor $\gamma$ in microglia/macrophages," Annals of Neurology, vol. 61, no. 4, pp. 352-362, 2007.

[104] X. Zhao, J. Grotta, N. Gonzales, and J. Aronowski, "Hematoma resolution as a therapeutic target: the role of microglia/ macrophages," Stroke, vol. 40, no. 3, pp. S92-S94, 2009. 


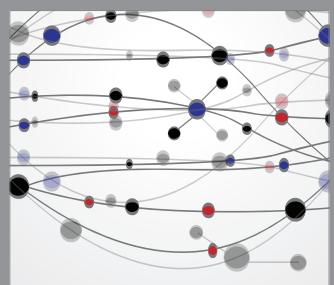

The Scientific World Journal
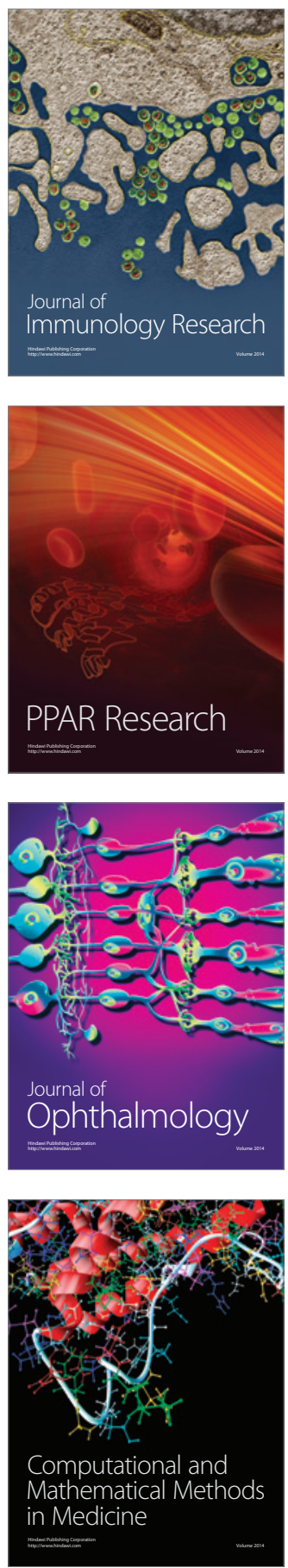

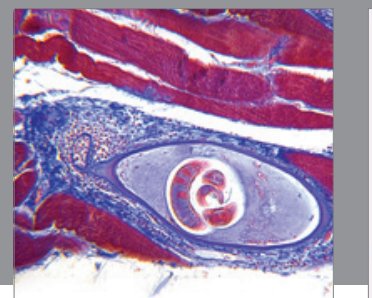

Gastroenterology

Research and Practice
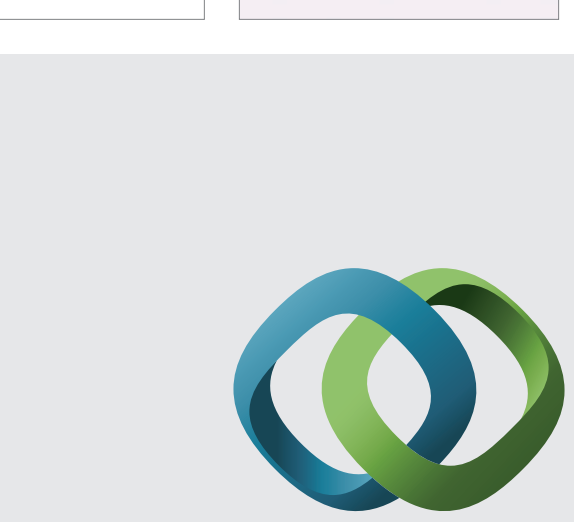

\section{Hindawi}

Submit your manuscripts at

http://www.hindawi.com
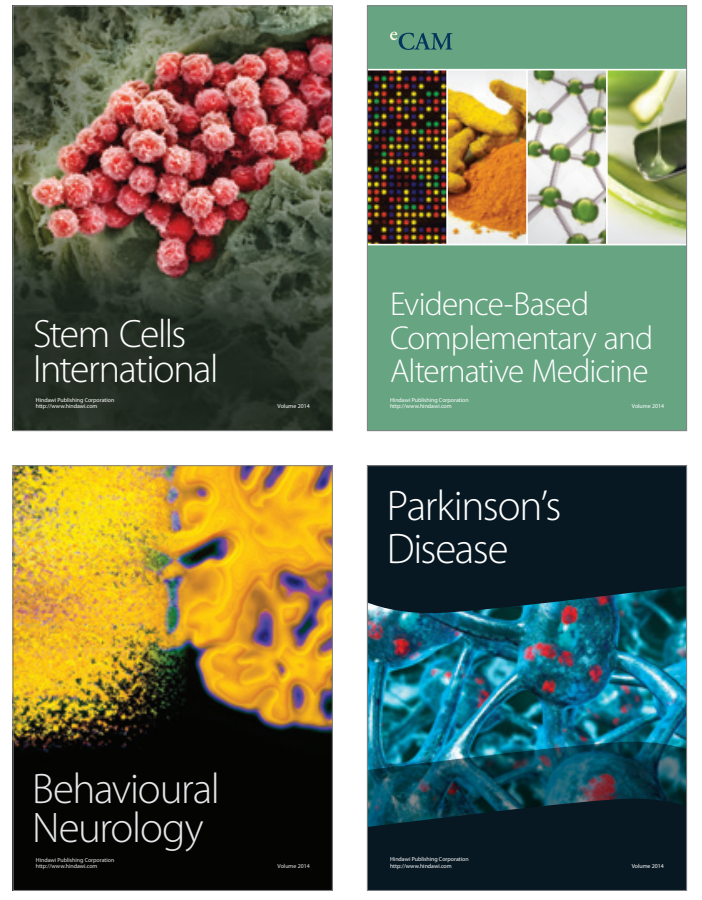
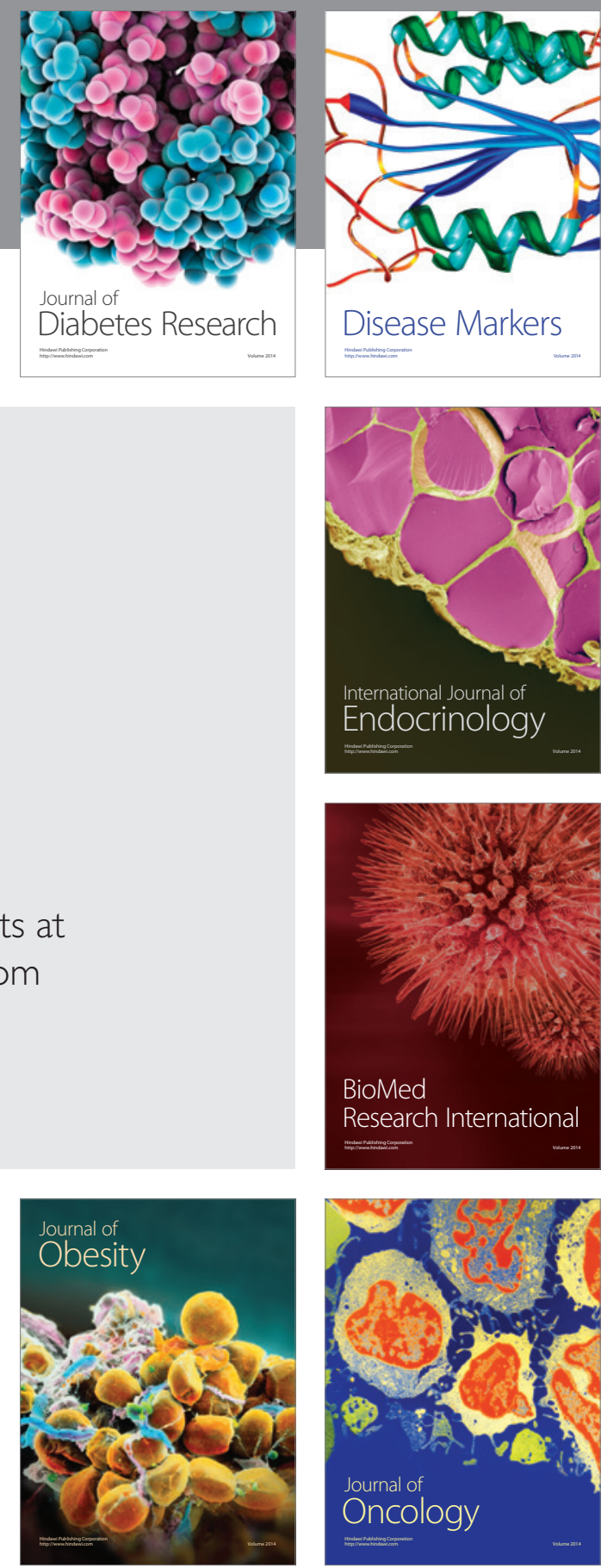

Disease Markers
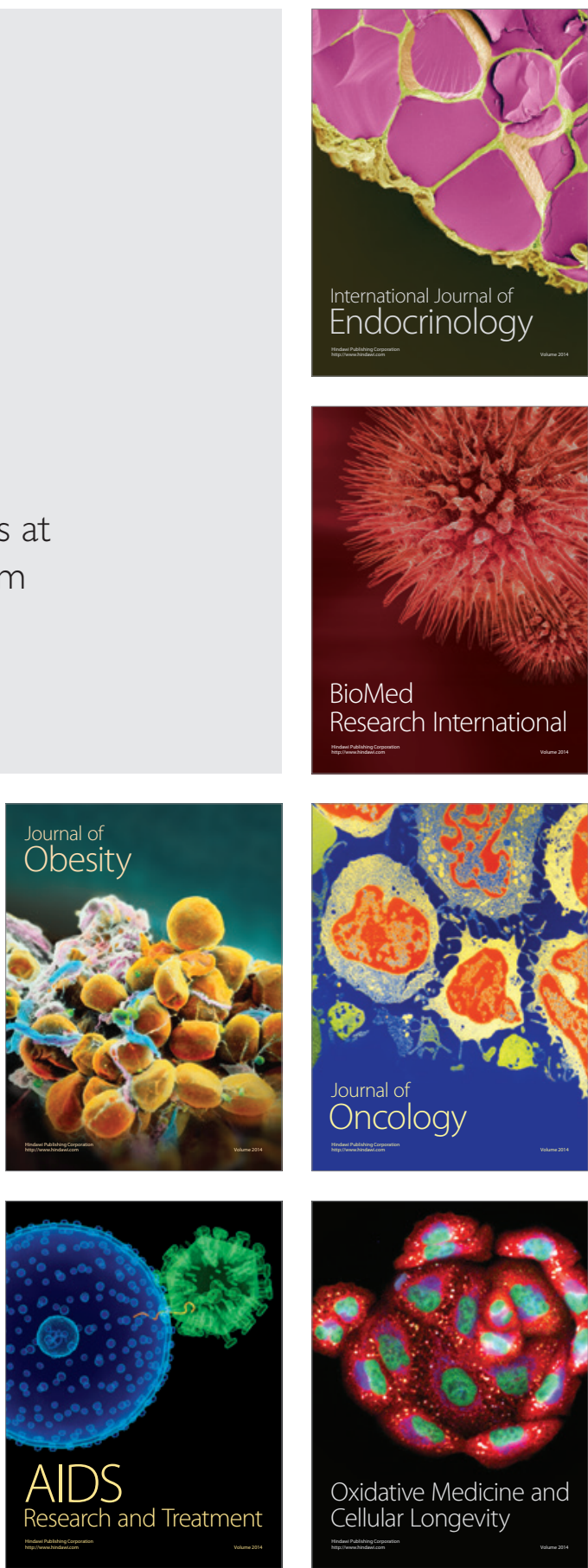